\title{
Invasive Electrographic Recording Techniques in Temporal Lobe Epilepsy
}

\author{
F. Dubeau and Richard S. McLachlan
}

\begin{abstract}
The management of uncontrolled partial epilepsy is a process dependent on a multidisciplinary and analytic approach. It is necessary to understand which lesions are epileptogenic, and if they are indeed responsible for the generation of seizures. In addition to localizing seizure onset, the functional and eloquent areas of the brain need to be identified. As in many other centres, we perform resective surgeries on the basis of combined information derived from seizure semiology, EEG abnormalities, neuroimaging and other tests of cerebral function. If surface EEG recording yields inconclusive or ambiguous results, then invasive intracranial techniques using intracerebral depth or subdural electrodes can be used to improve diagnostic or prognostic accuracy. The indications, principles, results and complications of these recording techniques based on extensive experience at two epilepsy surgery centres are reviewed.
\end{abstract}

RÉSUMÉ: Techniques effractives d'enregistrement électrographique dans l'épilepsie temporale. Le traitement des crises partielles non contrôlées est un processus qui dépend d'une approche multidisciplinaire et analytique. Nous devons comprendre quelles lésions sont épileptogènes et si ces lésions sont effectivement responsables des crises. Nous devons également déterminer les effets des crises sur le cerveau et identifier les zones fonctionnelles et éloquentes du cerveau. Comme dans bien d'autres centres, nous procédons à des résections chirurgicales basées sur l'information combinée de la séméiologie des crises, des anomalies de l'ÉEG, de la neuroimagerie ainsi que d'autres épreuves de fonction cérébrale. Si les résultats de l'ÉEG de surface sont non concluants ou ambigus, les techniques intracrâniennes invasives utilisant des électrodes intracérébrales profondes ou sous-durales peuvent être utilisées pour améliorer la précision du diagnostic et du prognostic. Nous revoyons les indications, les principes, les résultats et les complications de ces techniques d'enregistrement en nous basant sur l'expérience considérable de nos deux centres de chirurgie de l'épilepsie.

Can. J. Neurol. Sci. 2000; 27: Suppl. 1 - S29-S34

Surgery for epilepsy is considered when seizures are of sufficient frequency and severity to interfere with an individual's lifestyle. There is no particular seizure pattern that can be defined which qualifies a seizure patient for presurgical evaluation. The partial epilepsies are, however, typically the most medically intractable. Focal resections in patients with intractable epilepsies and well defined structural brain abnormalities identified as responsible for the generation of seizures have the best outcomes and lowest morbidity.

The prototype of a surgically remediable epileptic syndrome is mesial temporal lobe epilepsy (MTLE), which presents with a characteristic symptom complex indicating the presence of epileptic activity and an underlying lesion, usually hippocampal sclerosis. ${ }^{1}$ When history and seizure semiology suggest MTLE, if different antiepileptic drugs (AED) regimes do not completely control seizures, and if one is persuaded that no long remission may occur, then it becomes clear that the surgical option should be considered. At this point, definitive diagnosis and identification of the epileptogenic area can often be made by using noninvasive methods, based on the seizure type, interictal and ictal EEG features, neuropsychological and functional evaluation, and results of magnetic resonance imaging.

Some degree of unifocal electrical excitability is needed to identify the epileptogenic zone, ${ }^{2}$ and total resection of this area is necessary to stop the seizures. The consensus is that if one can document more or less precise EEG localization with noninvasive methods and if these findings are concordant with other means of demonstrating the mesial temporal anomalies, and not

From the Department of Neurology \& Neurosurgery, McGill University, Montreal Canada (F.D.) and the Department of Clinical Neurological Sciences, University of Western Ontario, London, Canada (R.S.M.)

Reprint requests to: R.S. McLachlan, London Health Sciences Centre, 339 Windermere Rd., London, Ontario, Canada N6A 5A5 
discordant with any, then there is no need for further investigation, particularly invasive EEG recording.

If presurgical surface EEG recording, with additional EEG electrodes (e.g. sphenoidal or mandibular notch electrodes) cannot provide an adequate answer, invasive intracranial techniques (primarily intracerebral or subdural electrode recordings) can be used to improve localization of the epileptogenic zone. ${ }^{3,4}$ Modern diagnostic technology has contributed to a better understanding of the pathophysiology of MTLE, and increased the ability to define precisely the epileptogenic zone and its relation with respect to the epileptogenic lesion in the temporal lobe. Recent studies have shown that qualitative and quantitative MRI (volumetric analysis) studies are highly sensitive and specific in detecting in vivo atrophy of the amygdala (Am) and hippocampus ( $\mathrm{Hc})$, and that those images correlate well with pathological substratum. . $^{5,7}$ The question remains, however, whether mesial temporal volume reduction or increased T2 signal in the mesial structures found by MRI correlate with mesial temporal seizure onset and outcome of epilepsy surgery. We are still debating if such focal MRI changes in Am and Hc alone or together are independent indicators of the results of other investigative modalities (clinical, EEG, and functional studies) used for diagnosis of MTLE. ${ }^{8,9}$ As neuroimaging and other localization techniques improve, there will be less need for invasive intracranial recordings, however, there will always remain a group of patients for whom such recordings remains necessary for accurate localization of the seizure focus.

\section{INDICATIONS FOR INVASIVE RECORDING}

In the past, the need for intracranial recording in the determination of an epileptogenic focus may have been overemphasized at some centres where invasive procedures, usually with depth electrodes, were required in all patients prior to epilepsy surgery. It is now recognized that in most cases of MTLE, standard electroencephalography combined with sphenoidal electrodes and evidence of mesial sclerosis on MRI, will suffice to define the surgical focus. When this does not occur, there are a wide range of indications to proceed with intracranial recording (Table 1). These are related to the crucial problems of lack of lateralization to one hemisphere and of localization within one hemisphere. It goes without saying that the most fundamental element of seizure localization is its lateralization to one hemisphere. An error of lateralization is the pitfall to be avoided at all costs and this uncertainty and ambiguity can sometimes be clarified only by intracranial recording. Although the progress in brain imaging may have

Table 1: Reasons for Invasive Recordings

1) seizures lateralized but not localized

2) seizures localized but not lateralized

3) seizures neither localized nor lateralized

4) seizure location discrepant with other data

5) relation of seizure localization to eloquent areas

6) relation of seizure localization to lesion
Table 2: Advantages and Disadvantages of Monitoring with Intracranial Electrodes in TLE

Advantages

- improved spatial resolution and increased sensitivity (early signal)

- no attenuation from scalp and skull

- no muscle artefact

- useful for direct electrical stimulation of cortex

\section{Disadvantages}

- limited cortical sampling or risk for sampling error (tunnel vision)

- not useful for functional cortical mapping (depth electrodes)

- risk of major complications (2-3\%)

already reduced the need for intracranial recording, at times it has created additional indications for this approach. There is still variation in the indications for, techniques of, and emphasis placed on invasive recording from centre to centre. The information in this report reflects the biases of two large and experienced epilepsy surgery units, one with a preference for subdural electrode recording and the other for the use of depth electrodes.

Intracranial recording provides accurate electrophysiological information used by the investigator to answer specific questions; as in the case of any other intracranial investigation, a well defined working hypothesis must be developed prior to undertaking such a study. This technique has been most useful for solving problems of lateralization and further localization in temporal lobe epilepsy. For instance, it is used to define seizure onset when extracranial localization is unclear in: 1) bilateral temporal epilepsy, 2) mesial versus neocortical temporal epilepsy, 3) mesial temporal versus orbitofrontal or cingulate gyrus seizure, and 4) occipito-temporal epilepsy. Any temporal lobe syndrome characterized by the presence of bilateral interictal or ictal anomalies arising from both temporal areas, the occurrence of a seizure interpreted as originating from the side contralateral to the maximal interictal or MRI abnormalities, or the recording of seizures with undetermined side of onset warrants further investigation with subdural or depth electrode recording.

\section{PrinCiPLES OF INTRACRANIAL RECORdING}

There are a number of technical advantages and disadvantages to recording with depth or subdural electrodes (Table 2). The main purpose of intracranial recording is to further delineate the area of onset and early propagation of a seizure. It is therefore important to cover the suspected zone of onset by placing electrodes in strategic areas, and to add specific electrodes when the clinical pattern or EEG data suggest another region as the potential site of onset. The idea is to confirm that seizures arise in one area and not in another.

One must keep in mind the physiological and biophysical principles which determine what signal is recorded by different types of electrodes. ${ }^{10,11}$ The generators of the EEG are convoluted dipole layers and the potential at any electrode, 


\section{Electronic permission not granted}

Figure 1: Left: an intracerebral depth or subdural electrode in close contact with cortex $(\mathrm{Cx})$ records a spike from a small cortical generator subtended by a fairly large solid angle.

Right: scalp electrode records a potential of the same amplitude from a much larger area of cortex subtended by a solid angle of the same size (from Reference 11, with permission).

whether inside or outside the head, is directly proportional to the solid angle which the dipole layer, acting as a generator of a signal, subtends at the electrode site (Figure 1). A scalp electrode picks up activity generated from a large cortical surface of approximately six square centimetres whereas subdural or depth electrodes will detect potential changes which occur over only a few square millimetres or less of cortex. Thus, depth electrodes are more sensitive in detecting spikes and seizures from a localized area but may miss epileptiform activity which occurs in adjacent regions only a short distance away. The electrographic picture obtained with invasive depth electrodes can be dominated by small and possibly clinically insignificant generators of epileptiform activity with which they are in direct contact, to the detriment of larger and often only slightly more remote generators. In contrast, many spikes recorded by depth electrodes appear diffusely in many contacts making it difficult to distinguish between propagated and locally generated discharges. For these reasons, interictal spikes recorded from depth electrodes can be unreliable indicators of seizure origin and hence, of the epileptogenic zone. The subdural electrode is somewhat of a compromise between scalp and depth electrode recordings of interictal spike activity but is more comparable to the former in delineating the putatative seizure focus. The value of ictal EEGs is greater than that of the interictal recording using both subdural and depth electrodes. Variations in the pattern of ictal onset (eg. repetitive spikes, low voltage, recruiting rhythm, rhythmic augmenting slow waves), in the propagation time and in the spatial distribution of seizures are all important variables that need to be considered in the interpretation of EEG signals during an ictal event.

\section{Complications}

The use of invasive subdural or depth electrode recording is not without potential complications (Table 3). Patients are more likely to experience some degree of headache, nausea and malaise related to meningeal irritation with subdural compared to depth electrodes but these are easily treated with analgesics. Infection occurs in $1-2 \%$ of patients and can require early removal of the electrodes. Such infections are usually minor but rare intracerebral abscesses have occurred. An unanswered question is whether the use of invasive subdural or depth electrodes increases the risk of infection following subsequent temporal resection. Empirical observations at our centres suggest that this is not a problem. Hemorrhage is rare and less likely to occur with subdural compared to depth electrodes. Although the penetrating intracerebral tracks made by depth electrodes and occasionally subdural electrodes do create surrounding gliosis, there is no evidence that these are epileptogenic.

\section{Electical STIMUlation}

Both subdural and depth electrodes can be used for electrical stimulation studies to determine relative irritability of cortex under different electrodes by the propensity for local after discharge or seizure generation following stimulation. However, subdural electrodes are more useful than depth electrodes to identify areas of eloquent cortex such as those involved in language or motor function by electrical stimulation or recording evoked potentials. Many empirical observations have been made in this regard but few studies have been published to address the usefulness of stimulating through invasive electrodes, particularly in the temporal lobe.

\section{Subdural electrodes - the London Health Sciences Centre series}

A recording technique which is less invasive to the brain than penetrating depth electrodes is the use of electrodes placed in the subdural space. The first description of the use of subdural electrodes to delineate seizure foci was by Herbert Jasper in two patients, JE and DTR in $1954 .{ }^{12}$ Thirty years later, other reports of the use of subdural strip electrodes began to emerge. ${ }^{13,14}$ Studies at some centres have suggested that in comparison to depth electrodes, subdural electrode recordings from the temporal lobe are equally effective in the detection of interictal spikes but fall short in accurately localizing seizures. ${ }^{15,16,17}$ Part of the reason for this may relate to drawbacks to the type of

Table 3: Complications of Invasive Electrode Recordings

- aseptic meningitis

- cerebrospinal fluid leak

- cerebral edema

- brain contusion

- scalp infection

- contribution to post-resection infection?

- subdural bleeding

- intracerebral hemorrhage 


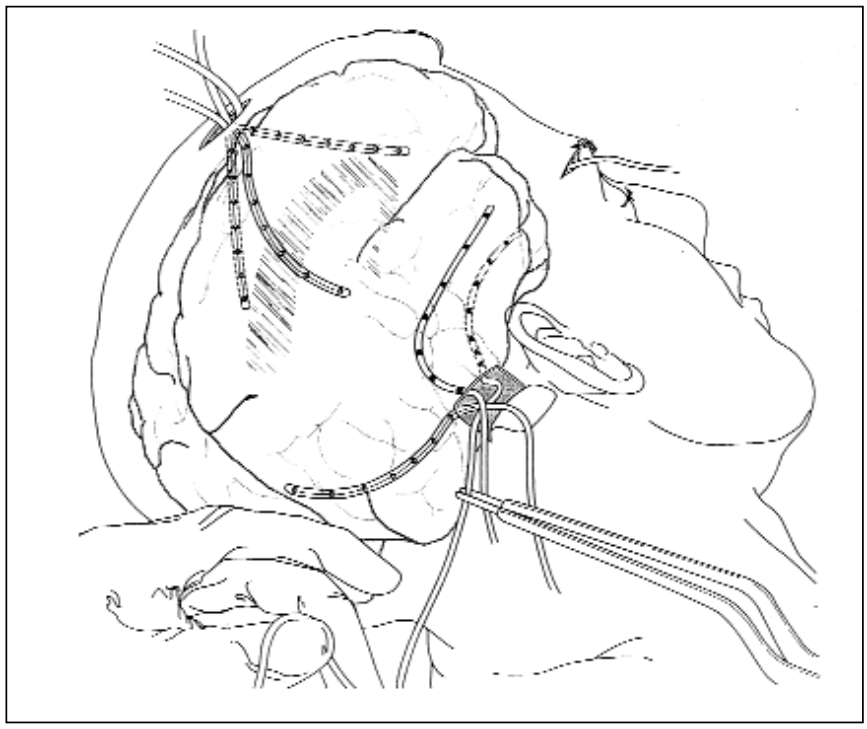

Figure 2: Two of three 7-contact tubular silastic subdural electrodes inserted through a posterior temporal burr hole to cover the temporal lobe are shown. All temporal and extratemporal cortical surfaces can be covered using three burr holes (courtesy of Dr. R. Sahjpaul).

flattened strip electrodes popularized by Wyler ${ }^{13}$ and the way these are surgically implanted for temporal lobe studies. The orthogonal insertion of these electrodes from lateral temporal burr holes allows limited coverage of the temporal lobe, particularly the mesial structures where seizures usually arise.

At London Health Sciences Centre, we have designed our own tubular subdural electrode utilizing seven stainless steel concentric rings, $10-20 \mathrm{~mm}$ apart, embedded in silastic tubing. For bitemporal lobe cases, three lines of electrodes are inserted through a posterior temporal burr hole, giving excellent full coverage of the mesial, inferior and convexity surfaces of the temporal lobe (Figure 2) and thus obviating the need for recording with depth electrodes in these cases. Average recording time is 2-3 weeks with a range of less than one week to eight weeks. During five years, from 1992-1996, 64 (31\%) of 209 temporal lobectomies were carried out after investigation with subdural electrodes. This compares to $57 \%$ of extratemporal resections which were done after subdural electrode recordings. Of the total number of 181 subdural implantations, both temporal and extratemporal, $124(66 \%)$ resulted in subsequent surgical resection of a seizure focus. In London, the use of subdural grids with up to 64 electrodes placed through a craniotomy under general anesthesia is largely confined to extratemporal cases, particularly those involving the Rolandic region.

It is rare that subdural recordings do not provide sufficient information in MTLE to test the hypothesis being addressed. Compared to scalp recordings, there is considerable improvement in seizure localization. Some ictal activity which cannot be appreciated at all on the scalp, such as that in simple partial seizures ${ }^{18}$ or rapid secondary generalization from a focus, is well recorded from subdural electrodes. Figure 3 shows the onset of a complex partial seizure which cannot be localized on
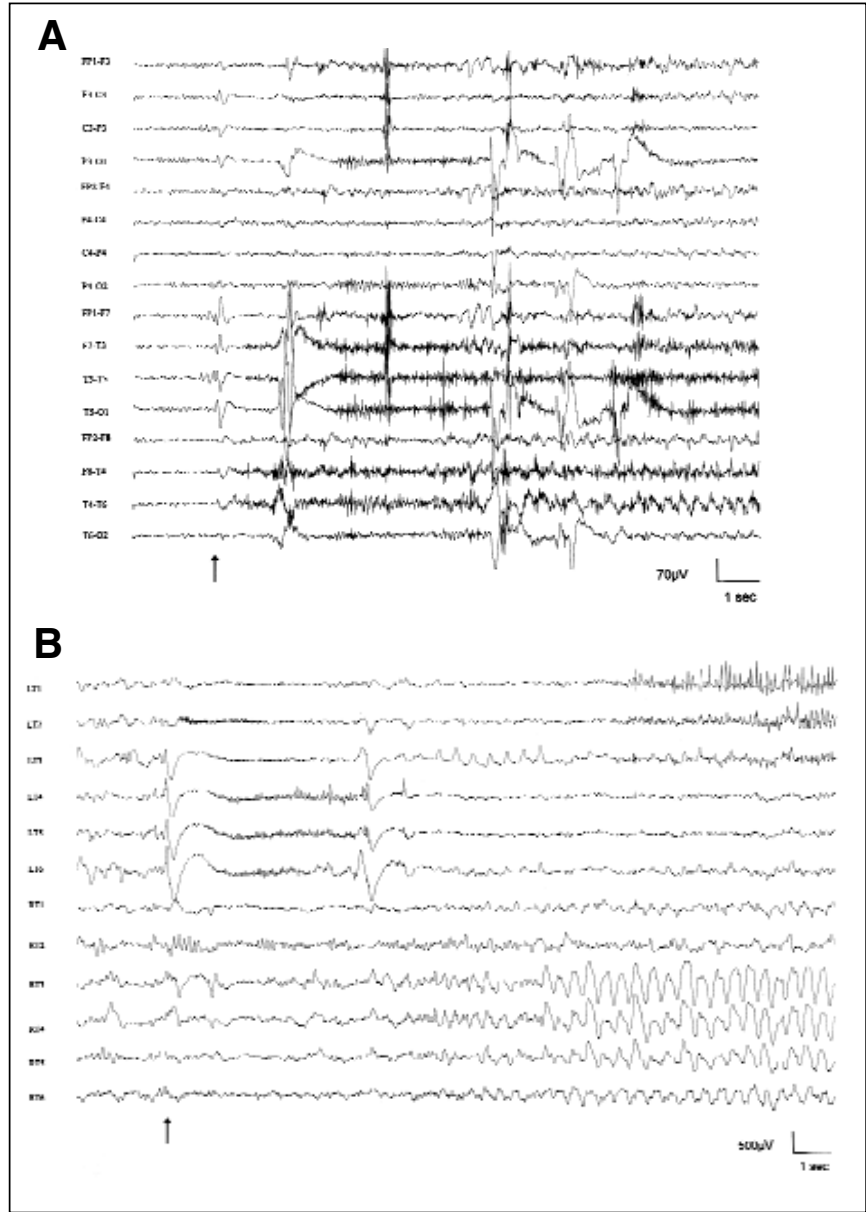

Figure 3: A) Onset of complex partial seizure (arrow) using scalp recording is obscured by muscle and movement artefact. Note initial left temporal spike followed after a few seconds by rhythmic right temporal waves. B) Subdural electrode recording clearly demonstrates low voltage, high frequency ictal onset from the left temporal lobe with subsequent propagation to right temporal lobe as rhythmic slow waves. $(L T=$ left temporal; $R T=$ right temporal $)$.

scalp recording but clearly starts in the left temporal lobe using subdural recording. Artefacts are somewhat greater than with depth electrodes, but considerably less than with scalp recordings and result mainly from movement particularly early in the recording. Other limitations include iatrogenic electrographic or clinical seizures caused by cortical contusions (this is a potential problem with depth electrodes as well) and poor resolution of epileptiform or background activity which has a wide regional or generalized field. For example, generalized spike wave can be difficult to recognize when recorded by subdural electrodes.

\section{Depth electrodes - the Montreal Neurological Hospital and Institute (MNH/MNI) series}

During the past three years, an average of 145 epileptic patients per year underwent prolonged video/EEG monitoring at the MNH/MNI. Mean duration of monitoring was nine days with an average number of ten (including electroencephalographic 


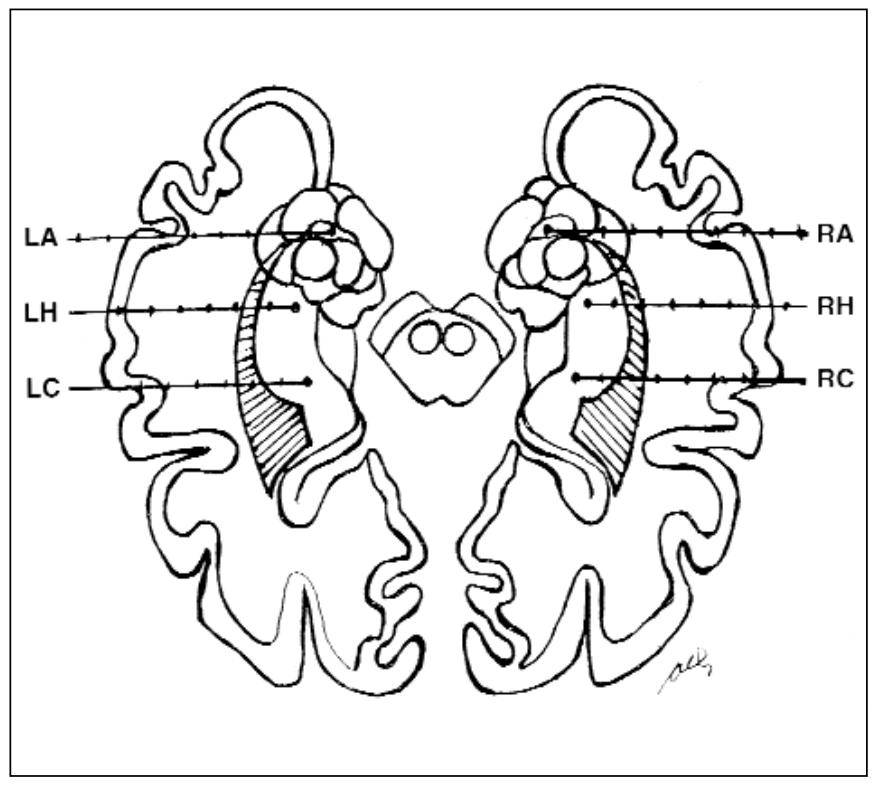

Figure 4: Typical depth electrode placement in amygdala (A), anterior hippocampus $(H)$ and posterior hippocampus $(C)$ for investigation of bitemporal epilepsy. $L=$ left, $R=$ right

attacks) episodes recorded in each patient. Every year, approximately 10-20 of these patients underwent intracranial depth electrode investigation when localization could not be clarified by extracranial EEG studies and detailed, noninvasive, structural and functional imaging tests.

\section{Patients}

The MNH/MNI series comprised two groups of patients with intractable TLE who had been investigated with depth electrodes between 1972 and 1986 (Group 1) and between 1987 and 1997 (Group 2). This division was somewhat arbitrary but represents two eras in the investigation and treatment of patients with MTLE: the pre- and post-MRI technology era. EEG computerization represents another major technical improvement that distinguishes the two periods. The results of stereotaxic depth electrode investigations (SEEG) in patients of the first group were reported by So et al in $1989 .{ }^{19}$

The complete series included a total of 137 patients with TLE, 57 in Group 1 and 80 in Group 2, which represents 57\% of the total number of patients investigated with SEEG technique, at the MNH/MNI, for 1972 through 1997. In Group 1, 57 patients with medically intractable seizures of presumed temporal lobe origin were investigated with SEEG because they had bilateral independent epileptiform discharges over the temporal regions as the major interictal epileptiform abnormality in extracranial EEG studies. Group 2 was more heterogeneous. As in the first group, 61 patients $(76 \%)$ were investigated with SEEG because they showed predominantly bilateral independent temporal epileptiform activity. In the remaining 19 patients, however, SEEG investigation was undertaken because of widespread bilateral temporal and extratemporal epileptiform discharges, or unilateral frontotemporal or temporo-occipital epileptic abnormalities (ten patients with unilateral depth electrodes implantation). The duration of recording averaged 18 days varying from eight to 30 days in Group 1 and six to 43 days in Group 2.

\section{Implantation methods}

Stereoelectroencephalography (SEEG) as initially developed by Talairach and Bancaud, ${ }^{20}$ has been further developed at the $\mathrm{MNH} / \mathrm{MNI}$ by the incorporation of digital subtraction angiography (DSA), MRI and PET in stereotactic brain mapping methods. Electrodes can now be directed precisely on the surface or within a gyrus or toward any deep structure. Intermediate recording sites can also be identified in relation to buried cortical areas. To refer to the use of "depth electrodes" is a misnomer since the electrodes provide a tridimensional evaluation of the seizure process, which necessitates placement of electrodes in deep, intermediate and superficial planes.

We use semiflexible, homemade tresses of stainless steel electrodes with nine contacts located $5 \mathrm{~mm}$ apart. Each contact forms a wire loop of $0.52 \mathrm{~mm}$ diameter and $0.1 \mathrm{~mm}$ thickness, adequately insulated to prevent artefacts. For the temporal lobes, two or three pairs of electrodes are inserted symmetrically through burr holes by an orthogonal approach (Figure 4). They penetrate the temporal lobe horizontally (from lateral temporal surface targeted toward the Am, anterior Hc and mid-portion of the Hc and parahippocampus gyrus at various anterior/posterior locations) and are inserted with a rigid introducer. The electrodes are fixed to the skull to prevent loosening and movement. While introduction was traditionally done with the help of a stereotaxic frame, it can now be carried out with frameless MRI-guided stereotaxy. Epidural electrodes are often placed over the temporal neocortical surface to provide additional and complementary information. Extratemporal electrodes are used when significant participation of extratemporal structures cannot be excluded. Valproic acid may increase surgical bleeding due to quantitative thrombocytopressive and functional defects in platelet aggregation, and may also decrease plasma coagulation factors. We now discontinue valproate at least two weeks before implantation. To minimize the risk of infection we do not use simultaneous extracranial EEG electrodes.

The guidelines for longterm monitoring of the SEEG are the same as for scalp recording. Initially, SEEG was recorded on 16 or 32 channels together with continuous video monitoring. Now, 64 channels of simultaneous recording are preferred. Maximal information is obtained through the newer methods with computerized assistance and multiple channel storage and reformatting. Spontaneous seizures with documented clinical symptoms or signs are studied including auras accompanied by ictal electrographic discharges. "Pure EEG seizures" and seizures precipitated by electrical stimulation are also analysed but their significance remains unclear for the moment. The principal epileptogenic zone is usually defined on a basis of the site (or sites) of seizure onset (ictal onset). Clinical activity preceding electrical seizure onset may indicate that the depth electrodes were recording from some distance away from the epileptogenic zone. Ictal onsets are classified as: 1) focal temporal when the seizure discharge originates in one anatomical area within the temporal lobe, eg. the hippocampus; 2) regional temporal when the ictal discharge starts simultaneously in more than two adjacent anatomical areas, eg. the hippocampus and the amygdala or the limbic and neocortical 
structures; 3) extratemporal; 4) widespread unilateral, eg. frontotemporal onset; and 5) bilateral temporal or diffuse.

Surface and depth EEG findings in patients with hippocampal sclerosis were recently compared with MR imaging. ${ }^{6,7,21}$ One of the important problems is to understand the role of Hc atrophy in the group of intractable epilepsy patients considered for possible surgery. Very few studies have examined the role of SEEG in the evaluation and interpretation of $\mathrm{Hc}$ atrophy in patients with MTLE. Our results seem to suggest a good but not perfect concordance between MRI detection of Hc atrophy and SEEG ictal localization of epileptogenic zone. For instance, in a small but significant number of patients, discordant results of SEEG and Hc atrophy were found. Some patients underwent surgery on the nonatrophic site with excellent results, while others had a poor outcome.

The significance of background abnormalities recorded from depth electrodes remains unclear and even has been ignored in most studies. In a recent study, we found no correlation between the frequency of SEEG interictal spikes recorded from mesial temporal structures and the amount of Am-Hc atrophy. ${ }^{8}$ There was, however, a significant correlation between background abnormalities and Am-Hc atrophy. This may suggest that continuous polymorphic slow waves and decrease or loss of normal fast rhythms are a better indicator of structural epileptogenic damage in mesial temporal structures than the frequency of interictal epileptiform discharges.

\section{REFERENCES}

1. Engel J, Jr. Update on surgical treatment of the epilepsies: Summary of the second international Palm Desert conference on the surgical treatment of the epilepsies (1992). Neurology 1993;43:1612.

2. Lüders H, Awad I. Conceptual considerations. In: Lüders H (ed): Epilepsy Surgery. New York: Raven Press, 1992:51-62.

3. Ojemann GA, Engel J. Acute and chronic intracranial recording and stimulation. In: Engel J, Jr (ed): Surgical Treatment of the Epilepsies. New York: Raven Press, 1987:263-288.

4. Spencer SS, Sperling MR, Shewmon DA. Intracranial electrodes. In: Epilepsy: A Comprehensive Textbook. Engel J and Pedley TA (eds). Philadelphia, Lippincott-Raven Publishers, 1998:1719-47.

5. Cascino GD, Jack CR Jr, Parisi JE, et al. Magnetic resonance imaging-based volume studies in temporal lobe epilepsy: pathological correlations. Ann Neurol 1991;30:31-36.
6. Jack CR Jr, Sharbrough FW, Cascino GD, et al. Magnetic resonance image-based hippocampal volumetry: correlation with outcome after temporal lobectomy. Ann Neurol 1992;31:138-146.

7. Cendes F, Andermann F, Gloor P, et al. MRI volumetric measurement of amygdala and hippocampus in temporal lobe epilepsy. Neurology 1993;43:719-725.

8. Cendes F, Dubeau F, Andermann F, et al. Significance of mesial temporal atrophy in relation to intracranial ictal and interictal stereo EEG abnormalities. Brain 1996;119:1317-1326.

9. King D, Spencer SS, McCarthy G, Spencer DD. Surface and depth EEG findings in patients with hippocampal atrophy. Neurology 1997; 48:1363-1367.

10. Gloor P. Neuronal generators and the problem of localization in electroencephalography: application of volume conductor theory to electroencephalography. J Clin Neurophysiol 1985;2:327-354.

11. Gloor P. Electroencephalography and the role of intracerebral depth electrode recordings in the selection of patients for surgical treatment of epilepsy. In: Porter RJ, Mattson R, Ward AA, Dam $\mathrm{M}$, eds. Advances in Epileptology: XVth Epilepsy International Symposium. New York: Raven, 1984:433-437.

12. Penfield W, Jasper H. Automatism, amnesia and mental disturbance. In: Epilepsy and the Functional Anatomy of the Human Brain. Boston: Little, Brown and Co., 1954:517-519.

13. Wyler AR, Ojemann GA, Lettich E, Ward AA. Subdural strip electrodes for localizing epileptogenic foci. J Neurosurg 1984;60: 1195-1200.

14. Rosenbaum TJ and Laxer KD. Subdural electrode recordings for seizure focus localization. J Epilepsy 1989;2:129-135.

15. Spencer SS, Spencer DD, Williams PD, Mattson R. Combined depth and subdural electrode investigation in uncontrolled epilepsy. Neurology 1990;40:747-9.

16. Sperling MR, O'Connor MJ. Comparison of depth and subdural electrodes in recording temporal lobe seizures. Neurology 1989;39: 1497-1504.

17. Brekelmans GJF, van Emde Boas W, Velis DN, et al. Comparison of combined versus subdural or intracerebral electrodes along in presurgical focus lateralization. Epilepsia 1998;39:1290-1301.

18. Devinsky O, Sato S, Kufta CV, et al. Electroencephalographic studies of simple partial seizures with subdural electrode recordings. Neurology 1989;39:527-533.

19. So NK, Gloor P, Quesney LF, et al. Depth electrode investigations in patients with bitemporal epileptiform abnormalities. Ann Neurol 1989;25:423-431.

20. Talairach J, Bancaud J. Stereotaxic approach to epilepsy. Prog Neurol Surg 1973;5:297.

21. Cascino GD, Trenerry MR, Sharbrough FW, et al. Depth electrode studies in temporal lobe epilepsy: relation to quantitative magnetic resonance imaging and operative outcome. Epilepsia $1995 ; 36: 230-235$ 